\title{
Real-time multi-marker measurement of organic compounds in human breath: towards fingerprinting breath
}

Running title: Real-time multi-marker measurement of breath

\author{
Iain R White ${ }^{1}$, Kerry A Willis ${ }^{1}$, Chris Whyte ${ }^{1}$, Rebecca Cordell ${ }^{1}$, Robert S \\ Blake $^{1}$, Andrew J Wardlaw ${ }^{2}$, Jonathan Grigg, ${ }^{3}$ Andrew M Ellis ${ }^{1}$ and Paul S \\ Monks $^{1 *}$ \\ ${ }^{1}$ Department of Chemistry, University of Leicester, Leicester, LE1 7RH, UK \\ ${ }^{2}$ Institute for Lung Health, Department of Infection, Immunity and Inflammation, \\ University of Leicester, Glenfield Hospital, Leicester, UK \\ ${ }^{3}$ Blizard Institute, Queen Mary University of London, 4 Newark Street, London \\ E12AT
}

*E-mail: P.S.Monks@1eicester.ac.uk

\begin{abstract}
The prospects for exploiting Proton Transfer Reaction-Time of Flight-Mass Spectrometry (PTR-ToF-MS) in medical diagnostics are illustrated through a series of case studies. Measurements of acetone levels in the breath of 68 healthy people are presented along with a longitudinal study of a single person over a period of one month. The median acetone concentration across the population was $484 \mathrm{ppbV}$ with a geometric standard deviation (GSD) of 1.6, whilst the average GSD during the single subject longtitudinal study was 1.5 . An additional case study is presented which highlights the potential of PTR-ToF-MS in pharmacokinetic studies, based upon the analysis of online breath samples of a person following the consumption of ethanol. PTR-ToF-MS comes into its own when information across a wide mass range is required, particularly when such information must be gathered in a short time during a breathing cycle. To illustrate this property, multicomponent breath analysis in a small study of cystic fibrosis patients is detailed, which provides tentative evidence that online PTR-ToF-MS analysis of tidal breath can distinguish between active infection and non-infected patients.
\end{abstract}




\section{Introduction}

Human breath measurements have a long history of aiding physicians in their diagnosis of illness and ailments. For example, physiological measurements of lung function, as assessed by spirometry, are used to screen general respiratory health in a manner analogous to that of blood pressure in cardiovascular health. These tests are inherently non-invasive; a significant advantage to their use in the clinical environment. However, spirometry alone may not yield the clinical aetiology of a disorder (Miller et al., 2005). There is, however, a potential wealth of information contained within the relative concentrations of the components of exhaled breath. Carbon dioxide measurements alone (as the third most abundant gas in exhaled air after nitrogen and oxygen) can provide information on alveolar ventilation and, indirectly, circulation and metabolic status. Increased $\mathrm{CO}_{2}$ production is observed in patients suffering, for example, from sepsis or tetanus. Another important example of a commonly measured endogenous compound is nitric oxide, which is increased in subjects with airway inflammation and its measurement is one of the few breath tests that have been developed for use in clinical practice (Dweik et al., 2011). After $\mathrm{CO}_{2}$ and the aforementioned gases, it is only water and a small number of inert gases that are present in appreciable quantities in breath, bringing the sum of individual components close to $100 \%$.

The small fraction that remains comprises a vast number of trace compounds; largely volatile organic compounds (VOCs) whose concentration can range from parts-per-million to the part-per-trillion by volume (ppmV to pptV). Just as capnography directly monitors exhaled $\mathrm{CO}_{2}$ and indirectly monitors $\mathrm{CO}_{2}$ present in arterial blood, VOCs detected in breath include those that have been produced in the body and are transported via the bloodstream to the lungs. It has been known for many years that breath odour can be an indication of disease, a common example being the characteristic fruity smell of the breath of diabetic patients due to high levels of acetone in the body (Tassopoulos et al., 1969).

On the basis that VOCs in breath are representative of VOCs in the blood and therefore processes occurring in the body, analysis of exhaled breath may become a non-invasive prognostic or diagnostic for use in clinical practice (Kim et al., 2012). With a wide range of VOCs present in breath, a variety of different analytical techniques have been used to measure them. Highly sensitive instruments are required to determine breath VOCs that might be present in concentrations as low as the pptV range. Gas chromatography - mass spectrometry (GC-MS) has in the past been the most commonly used technique (e.g. Jansson, 1969; Phillips, 1999a and 1999b) and has identified a large number of endogenous VOCs (Mieksch et al, 2004). The main disadvantage of GC-based systems is that sample pre-treatment (pre-concentration on sorbent traps or by solid phase microextraction) precludes on-line breath analysis (Boots et al., 2012). Over time, other techniques have been applied to breath research 
including ion-mobility spectrometry (IMS) (Baumbach, 2009; Ruzsany and Baumbach, 2005), selected-ion-flow-tube mass spectrometry (SIFT-MS) (Smith and Spanel, 2004) and proton-transferreaction mass spectrometry (PTR-MS) (Lindinger et al., 1998). SIFT-MS and PTR-MS are examples of direct MS techniques, exhibiting high sensitivities for VOC measurements and therefore near realtime measurements are made possible in both techniques (no sample pre-treatment is necessary) (Blake et al., 2009).

In general, most breath biomarker research to date has been conducted using the mixed air sampling technique due to its simplicity, cost and ease of use for patients (Steeghs et al., 2007). This approach generally utilises sampling bags, adsorbent tubes or canisters to collect whole breath samples. However, this approach is liable to contamination and dilution by dead-space air (Beauchamp et al. 2008). Time-controlled offline sampling aims to alleviate this but problems can occur due to physiological variation in dead-space volume and breathing patterns (Amann et al., 2010). In general, alveolar (end-tidal) air has the lowest concentrations of exogenous species and thus the advantage of employing direct online sampling is clear. However, the analytical technique employed needs to be fast and sensitive in order to be selective in the portion of the breath that is sampled. At present, there is no standardised approach to online breath sampling with direct MS but the established methods employed in spirometry, capnography and NO testing may offer a means to standardise breath VOC sampling and analysis (O'Hara et al., 2008) and recent progress in sampling techniques has resulted in the use of buffered end tidal sampling (Herbig et al. 2008).

To date, direct MS techniques have been applied to online breath measurements for various different systems and studies (Amann et al, 2004; Spanel and Smith, 2011) in order to identify biomarkers. Recently, Herbig et al. (2009) described a PTR-ToF-MS system capable of measuring multiple metabolite signals over a timeframe fast enough to characterise a full breath cycle. An advantage to a system such as this is that a full mass spectrum can be obtained for end-tidal breath so that multiple biomarkers can be identified and recorded. As breath samples represent a complex and unique mixture of gases, it is likely that multiple biomarkers best represent an individual's metabolic state. Furthermore, the benefit of using PTR-ToF-MS (compared to, for example, PTR-quad-MS) is not only its ability to rapidly capture entire mass spectra but also the resolving power that ToF analysers can exhibit, which may lead to more confident species assignment through accurate mass detection (Jordan et al, 2009, Blake et al, 2012).

The ability of PTR-ToF-MS to capture an entire mass spectrum (with potentially high resolution) under a fast rate of acquisition means that it may be possible to characterise pattern changes within mass spectra to characterise different populations. An analogous principle lies behind electronic noses, which are sensor arrays that are cheap, compact and easy to manufacture (Gardener et al., 
2000). As an example, electronic noses coupled with pattern recognition have been applied to lung cancer research (Di Natale et al., 2003; Dragonieri et al., 2007). Ultimately, a small, portable and simple instrument would be far more suited to everyday use in clinical practice than a costly and often large research instrument. At this early stage breath analysis research is likely to continue using specialised laboratory instruments, but in the longer term we anticipate that the findings from this research will lead to the construction of smaller instruments specifically designed for the detection of identified biomarkers. Technologies such as PTR-ToF-MS are well suited to breath biomarker research and discovery as they can be used to obtain complex spectra for pattern recognition and, following deconvolution, may yield information regarding the speciation of successfully identified biomarkers.

The present study demonstrates the versatility of PTR-ToF-MS as a tool for physiological and pharmacological studies and for medical diagnostics. These are illustrated using a series of case studies focused on both single biomarker measurements and multiple biomarker measurements. The single biomarker studies are aimed at quantifying acetone levels in human breath and measuring volatile metabolites following ethanol consumption. We then turn to the real strength of PTR-ToF-MS when compared with single channel mass spectrometric techniques, its ability to acquire the whole mass spectrum at once (in a rapid manner). Results are presented from a small-scale study of the VOCs in the breath of cystic fibrosis patients and we show that online breath sampling with PTRToF-MS has the potential to distinguish between infected and non-infected patients. 


\section{Materials and methods}

\subsection{Proton Transfer Reaction - Time of Flight - Mass Spectrometry}

The instrument used to generate the data presented in this study is described in detail by Blake et al. (2004) and Wyche et al. (2007). In brief, PTR-MS utilises chemical ionisation in the form of proton transfer, normally from $\mathrm{H}_{3} \mathrm{O}^{+}$, to ionise analyte molecules. The instrument described here is capable of using alternative reagent ions and so is sometimes referred to by the more general title of Chemical Ionisation Reaction (CIR)-MS. Chemical ionisation is a soft ionisation technique, as the reactions produce little excess energy. As a result, many product ions remain intact, although significant ion fragmentation is still observed for certain classes of compounds (Blake et al., 2009).

The production of relatively simple mass spectra makes PTR-MS an excellent technique for analysing complex VOC matrices such as breath. In addition, time-of-flight mass spectrometry brings with it the ability to rapidly monitor all mass channels simultaneously, providing a complete real-time VOC profile. During these experiments, in order to measure multichannel the ToF analyser was operated at a mass resolution $(\mathrm{m} / \Delta \mathrm{m})$ in excess of 1000 and was not utilised in high resolution mode. Key components of the instrument used include a radioactive ${ }^{241} \mathrm{Am}$ ion source and a drift tube coupled to a reflectron time-of-flight mass spectrometer (Blake et al, 2004). The drift tube of the PTR-ToF-MS instrument contains two regions. The main drift cell experiences a constant electric field to number density ratio $(E / N)$, whereas the small region between the last electrode and the exit aperture, which is referred to as the collision cell, can operate under electric field conditions different from the main part of the drift tube. The collision cell is normally used to promote fragmentation of hydrated VOC cluster ions. During breath sampling, the breath air is continuously introduced into the upstream end of the drift tube at a flow rate of $220 \mathrm{sccm}$, which is operated at a pressure of $6 \mathrm{mbar}$. A continuous flow of water vapour enters the top of the ion source at a set flow rate of $30 \mathrm{sccm}$. An $E / N$ of $80 \mathrm{Td}$ was used for the main part of the drift tube and $174 \mathrm{Td}$ for the collision cell.

\subsection{Single breath sampling (FVC-full vital capacity) at Glenfield Hospital and during the ethanol experiments}

For human breath measurements an end-tidal sampling scheme was employed. As will be detailed in later sections, this approach was used for both measurements of single molecules in breath during a study at Glenfield hospital (Leicester, UK) and for a study into the composition of exhaled breath following the consumption of ethanol. The breath sampling apparatus consisted of a plastic mouthpiece and an adult electrostatic bacterial/viral filter (Flexicare Medical Limited, Mountain Ash, 
Mid Glamorgan, UK) connected to a Digital Volume Transducer (DVT; VMM-400 Ventilation Measurement Module from Interface Associates, Laguna Niguel, CA, USA). The $15 \mathrm{~mm}$ outer diameter of the cartridge connected directly into the breath sampling apparatus. A T-piece connector with two non-return valves (Intersurgical, Wokingham, Berkshire, UK) was used to allow the patient to breathe room air on inhalation. The sample line between the apparatus and the instrument was heated to $40{ }^{\circ} \mathrm{C}$ and a heated blanket (Infroheat Limited, Wolverhampton, UK) surrounded the entire sampling apparatus, also heated to $40{ }^{\circ} \mathrm{C}$. The dead volume of the apparatus was approximately 100 $\mathrm{mL}$. The volunteers performed a full inhalation followed by a vital capacity exhalation. Breath was sampled from 68 different people: 34 male and 34 female, with an average age of 45 (range 16 - 87) and BMI of 26.9 (range 18.5 - 47.9). A number of subjects were current smokers (10 patients) or exsmokers (5 patients) based on patient declaration. On initial inspection of acquired data, 1 second was the longest time over which ions could be integrated during a full vital capacity exhalation such that breath profiles could be traced. The signal at $m / z=59$, corresponding to protonated acetone, is consistently observed in breath samples and was used in this case to visually select the end-exhaled region of each breath (an example is presented later in Figure 4). This region was then used to average over all mass channels with a typical duration of 1 to 3 seconds during a full vital capacity (FVC) exhalation. Subjects provided three direct breath measurements and all breath and ambient air measurements were made using 1 second integration times over an $\mathrm{m} / z$ range of $12-154 \mathrm{Da}$. The study was approved by the Leicestershire Research Ethics Committee

The same apparatus and analytical method was used during the ethanol consumption experiment. Two healthy volunteers (1 male, age 30, BMI 24.1 and 1 female, age 25, BMI 20.3) were asked to give single vital capacity breaths. Five breath measurements were recorded over a half hour period prior to any alcohol consumption so that baseline levels could be obtained. The volunteers then consumed 2 units (approximately $20 \mathrm{~mL}$ ethanol) of alcohol over a 10 minute period. End tidal breaths were then collected immediately after and then at regular intervals over the following 3 hours. No food had been consumed in the 2 hours prior to the experiment and no other food or drink was consumed during the course of the experiment.

Measured count-rates were calibrated into absolute concentrations using a gas standard generator (Model 491, KinTek) fitted with a humidifier unit (see also Wyche et al, 2007). Variable concentrations were delivered at $100 \%$ relative humidity $\left(T=40{ }^{\circ} \mathrm{C}\right)$ for permeation tubes containing acetone, acetonitrile, dimethylsulfide, acetic acid, ethanol (VICI, UK) methanol and isoprene (KinTek). Corresponding limits of detection for 1 second acquisition at $2 \sigma$ precision (with normalised sensitivities in parentheses) for these compounds were $15 \mathrm{ppbV}$ acetone (98 ncps at $m / z 59), 1 \mathrm{ppbV}$ acetonitrile (95 ncps at $m / z$ 42), 2 ppbV dimethylsulfide (74 ncps at $m / z 63$ ), $23 \mathrm{ppbV}$ acetic acid (27 
ncps at $m / z 61), 2059 \mathrm{ppbV}$ ethanol (0.5 ncps), $9 \mathrm{ppbV}$ methanol (41 ncps at $\mathrm{m} / \mathrm{z} 33$ ) and $26 \mathrm{ppbV}$ isoprene (7 ncps at $m / z 69)$.

Figure 1 outlines a schematic of the breath sampling apparatus used in this study. Experiments were carried out to assess whether the bacterial filter or any other part of the sampling apparatus removes VOCs in the sampling process. Known concentrations of VOCs were passed through the breath sampling apparatus using a gas standards generator (Model 491, KinTek) and reference measurements were obtained by passing the same mixture through a standard line of PFA tubing under both dry and humid conditions. The sampling loss tests were conducted for the compounds acetone, acetonitrile, dimethylsulfide, acetic acid, methanol, isoprene and ammonia. No observable loss was observed for these gases under the conditions described within the precision of the PTR-ToF-MS technique $(<2 \%$ relative standard deviation (RSD)).

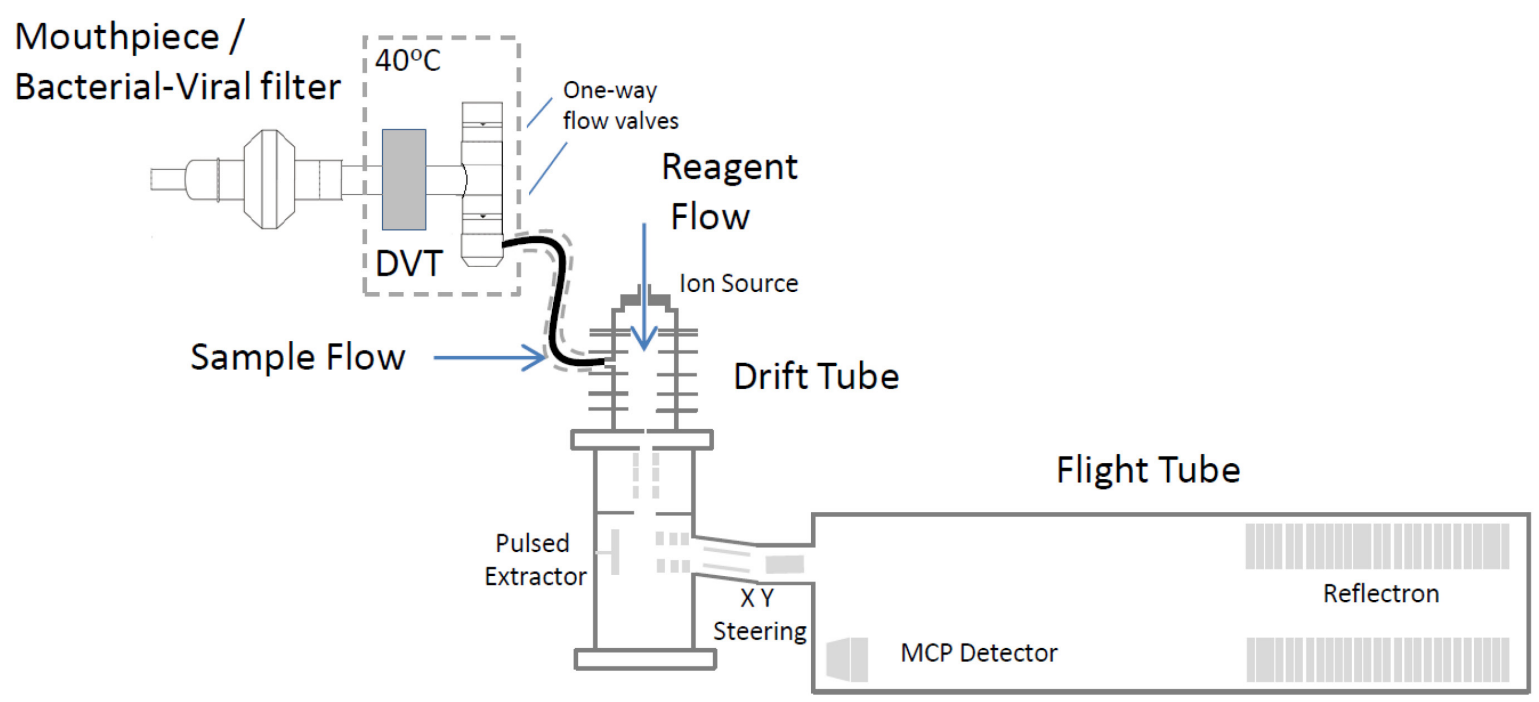

Figure 1. Schematic of the breath sampling apparatus coupled to PTR-ToF-MS (note that DVT $=$ Digital Volume Transducer and MCP = microchannel plate)

\subsection{Tidal breath sampling during the cystic fibrosis study}

The breath sampling apparatus used for the cystic fibrosis study was essentially the same as that for the studies described in the previous section, with a minor modification to the interconnecting tubing diameter which resulted in a dead volume of $170 \mathrm{sccm}$. Breath samples were collected from a small group of patients who were attending a cystic fibrosis clinic at Leicester Royal Infirmary. A total of 10 cystic fibrosis patients ( 5 male, 5 female, mean age 14) took part and 4 healthy children ( 3 male, 1 female, mean age 9) formed the control group. All cystic fibrosis subjects displayed chest symptoms 
at the time of the trial and were taking or had recently completed antibiotic treatment. Some subjects were additionally taking steroid-based anti-inflammatory medication and/or were receiving DNase treatment to reduce mucus viscosity. No subjects in the control group showed signs of illness at the time of the trial and were free of any chronic lung condition. In the cystic fibrosis group, sputum/cough swabs for culture were taken within 10 days prior to breath sampling: 5 cystic fibrosis patients were found to be infected (3 with Staphylococcus aureus, 2 with Aspergillus fumigatus, 1 with mixed flora). The study was approved by the Leicestershire Research Ethics Committee

Each patient was sat down for a few minutes to relax before sampling and 5 minutes of ambient air background measurements were made during this period. Breath collection involved tidal breathing, inhalation and exhalation via the mouth for 5 minutes. All patients were analysed during the afternoon, with no food having been consumed in the previous $2-3$ hours. Both ambient air and breath were recorded at 1 second integration times (typically $10^{4}$ complete mass scans) and a mass range up to $200 \mathrm{Da}$ was employed in the PTR-ToF-MS measurements. As during the sampling session breaths were continually captured, the signal to noise of a mass channel could be significantly increased if exhaled breath was integrated over multiple breaths thus improving the detection limit. The following detection limits were calculated over a timeframe of 1 minute and $2 \sigma$ precision: 3 ppbV acetone, $0.2 \mathrm{ppbV}$ acetonitrile, $0.2 \mathrm{ppbV}$ dimethylsulfide, $4 \mathrm{ppbV}$ acetic acid, $311 \mathrm{ppbV}$ ethanol, $1 \mathrm{ppbV}$ methanol and $3 \mathrm{ppbV}$ isoprene.

\section{Results and Discussion}

As ionisation conditions in PTR-MS can be soft, fragmentation is minimal for many compounds. Consequently, if a mass channel is free from nominally isobaric species the ion count rate at the specific $\mathrm{m} / \mathrm{z}$ reflects the concentration of the corresponding unprotonated species in the gas phase. In invoking this assumption, analysing single mass channels provides two main areas that can be exploited for research with direct breath measurements: single biomarker measurement (see section 3.2) and pharmacokinetic studies (see section 3.3). In PTR-MS, single biomarker analysis is particularly useful where a compound is both present at high concentration and is more sensitive towards PTR ionization when compared to any potential "interference" species. Where this is the case in breath, endogenous compounds can be measured in real-time and in certain cases it may be possible to relate them to a disease or disorder. Furthermore, to a first approximation, once identified, a biomarker can in principle be quantified theoretically using PTR-MS as long as the kinetics of ionisation are known and the reagent ion signal simultaneously recorded. In practice, this approach can lead to significant uncertainty (Blake et al, 2012). Additionally, the sensitivity of a species is dependent on sample humidity and $\mathrm{CO}_{2}$ content (though under these conditions the latter affect is negligible) (Keck et al., 2008). These issues can be explored and resolved by using external 
calibration to establish the concentration of a particular species (Wyche et al., 2007; Schwarz et al., 2009), which then makes it possible to reliably quantify individual compounds using PTR-MS.

\subsection{Real-time multi-channel measurements of breath}

To demonstrate the temporal and multichannel nature of breath VOC data from PTR-ToF-MS, Figure 2 shows an example following a typical data acquisition of a 5 minute tidal breathing exercise. This plot exemplifies the great strength of PTR-ToF-MS, i.e. the ability to monitor multiple compounds in real-time simultaneously. As shown most clearly in the upper trace, which focuses on acetone (protonated $\mathrm{m} / \mathrm{z}$ 59), the breathing cycle can be identified and thus alveolar breath can be discriminated from dead-space air by focusing on the appropriate sections of the breath trace. Clearly, since this time-resolved information is available in every mass channel simultaneously, a rich source of information is obtained.

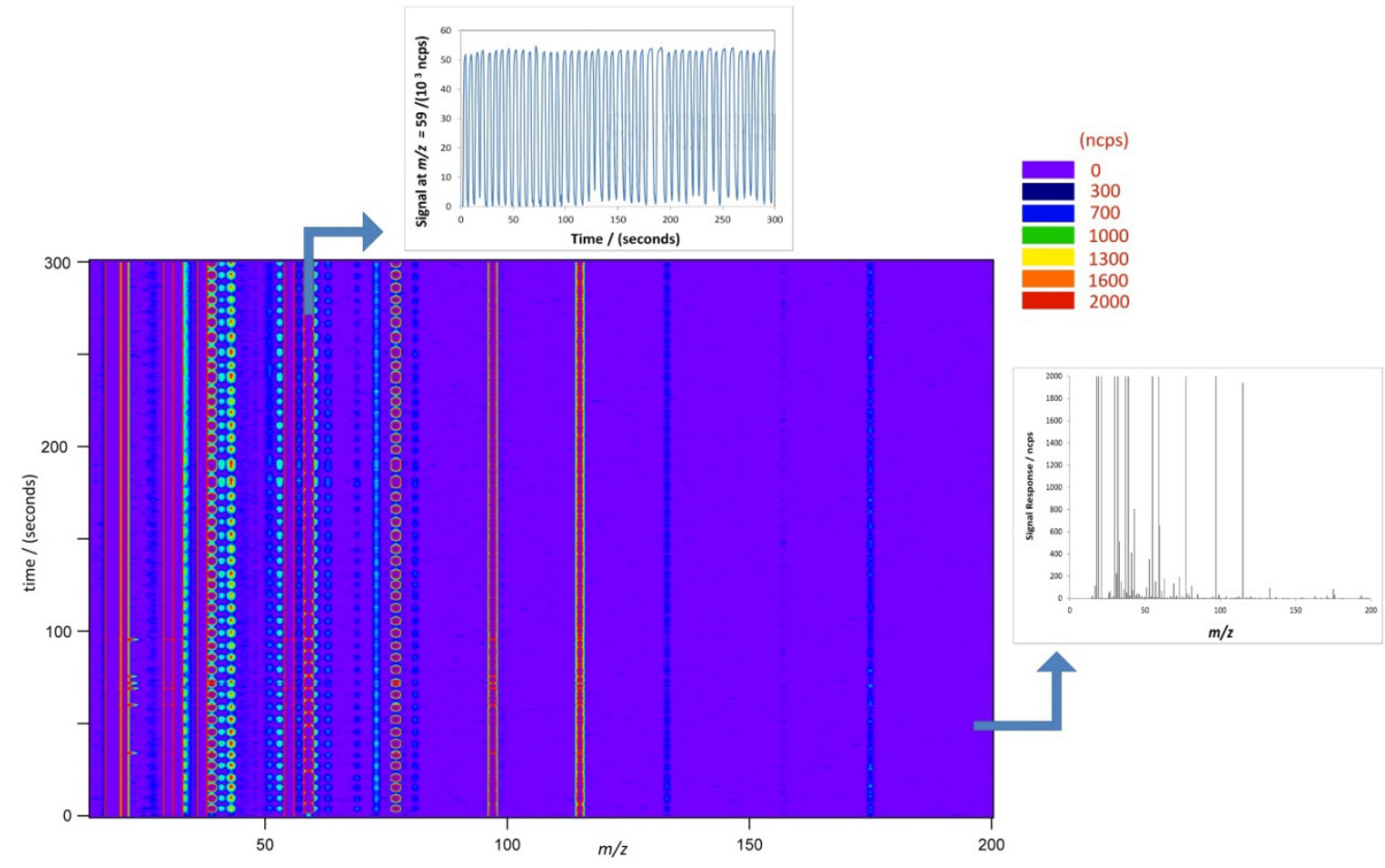

Figure 2.

Example breath analysis data output: 5 minutes tidal breathing degraded into a time series for a single ion at $m / z=59$ (top trace)). Also shown on the right hand side is a specific mass spectrum recorded at $t=50 \mathrm{~s}$. 


\subsection{Direct PTR-ToF-MS in single breath studies}

The quantitative performance of a technique is critical. Although multiple compound detection is where PTR-ToF-MS offers unique benefits, the technique can of course be used for exploring single compounds. An example of a species suitable for single compound analysis by PTR-MS is acetone, an ubiquitous breath component present in the high ppbV range and whose alveolar concentration varies over time and across populations (Turner et al., 2006; Smith et al. 2007). Figure 3 shows the distribution of acetone levels in the breath of a population of 68 subjects in a study conducted at Glenfield Hospital, UK, recorded using PTR-ToF-MS. In this study, patients provided three direct breath measurements, as described in section 2.2. The results obtained are indicative of those found elsewhere; for example, Smith and Spanel (2007) reported a population median of $477 \mathrm{ppbV}$ based on direct-breath results obtained from several studies using SIFT-MS, compared to $484 \mathrm{ppbV}$ (325 - 684 ppbV interquartile range (IQR); $163-4264$ ppbV total range) obtained here. Not only does this result support previous literature findings, the fact that these results are similar to those found using different analytical techniques adds credence to the concept of single compound analysis by direct PTR-ToF-MS. For example, Deng et al. (2004) used SPME-GC/MS to obtain a median acetone concentration of 520 (420 - 625 IQR; 220 - 800 total range) ppbV for 15 healthy controls whilst Schwarz et al. (2009) reported an acetone distribution with a median of $559 \mathrm{ppbV}$ and a total range of $280-1269 \mathrm{ppbV}$. It should also be noted that King et al. (2010) used PTR-MS in conjunction with GC-MS for unambiguous direct breath sampling of acetone.

Ueta et al. (2009), used in-needle pre-concentration and a GC-MS technique to find a total range of 190 to $660 \mathrm{ppbV}$ (270 to $550 \mathrm{ppbV} \mathrm{IQR)} \mathrm{for} \mathrm{acetone} \mathrm{in} \mathrm{a} \mathrm{controlled} \mathrm{type-II} \mathrm{diabetes} \mathrm{patient's} \mathrm{breath}$ (within the range found here for healthy controls). Uncontrolled type II diabetes resulting in ketoacidosis can cause elevated acetone levels in breath. Deng et al. reported higher values, between 1760 - 3730 ppbV (1955 - 2470 ppbV IQR), in 15 type II diabetics. For insulin-dependent diabetics, even whilst adequately controlled, breath acetone levels between 2000 - 5000 ppbV have been observed (Smith and Spanel, 1999). It is worthy of note that whilst the comparability of these results with our own highlights the quantitative nature of PTR-ToF-MS, the dynamic range of PTR-ToF-MS is such that it easily covers the spread in both healthy and diabetic breath acetone levels. In general, acetone population variability is likely to be caused by a number a factors including age, gender, BMI, physiology and illness.

The results from the Glenfield study (see methods section for details) are summarised in Table 1, alongside other compounds measured in the same study. Though species identities in Table 1 are tentative, peak assignment was supported by examining the ratio of the naturally occurring isotope peaks. 


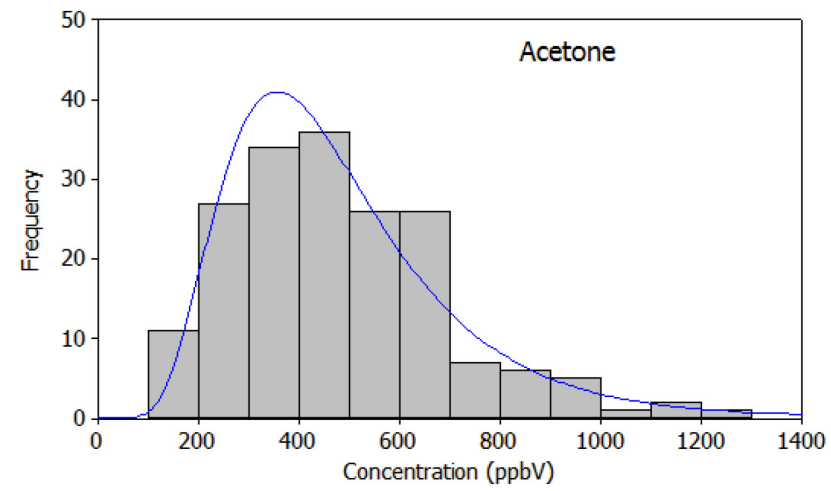

Figure 3. Population variability in acetone from direct PTR-ToF-MS measurements from the breath of 68 subjects using online (Full Vital Capacity) sampling. Data were acquired at $m / z=59$ (corresponding to protonated acetone ion) and employed $1 \mathrm{~s}$ signal integration.

Table 1. Median breath VOC levels obtained from the Glenfield study ( $\left.\mathrm{n}=68^{*}\right)$ compared to previous literature on healthy adult breath samples.

\begin{tabular}{cccl}
\hline Peak assignment & $\mathbf{m} / \boldsymbol{z}$ & \multicolumn{2}{c}{ Concentration in breath / ppbV } \\
This & Previous work \\
study & & 484 & $462^{\mathrm{a}}, 520^{\mathrm{b}}, 559^{\mathrm{c}}$ \\
\hline Acetone & 59 & 350 & $461^{\mathrm{a}}$ \\
Methanol & 33 & 193 & $106^{\mathrm{a}}, 240^{\mathrm{d}}$ \\
Isoprene & 69 & 2 & $<20^{\mathrm{e}}$ \\
Acetonitrile & 42 & 11 & $20-40^{\mathrm{f}}$ \\
Dimethyl sulfide $^{*}$ & 63 & & \\
\hline
\end{tabular}

*The acetonitrile concentration represents the median from a sample set of 49 non-smokers a) Smith et al. 2007, b) Deng et al. 2004, c) Schwarz et al, 2009, e) Abbott et al. 2003, f) Taucher et al. 1996

Acetonitrile, an exogenous compound commonly observed in the breath of smokers, is included in Table 1 based on the median of 49 declared non-smokers. As would be expected, in this study a significant difference $(p<0.05)$ was found between non-smokers (median $2 \mathrm{ppbV}, n=49$ ), exsmokers $(2 \mathrm{ppbV}, n=10)$ and smokers $(18 \mathrm{ppbV}, n=5)$. Four non-smoking subjects were excluded from the acetontrile dataset as their breath contained anomalously high levels of acetonitrile (possible reasons for this include inaccurate assignment of smoking status, the potential effects of secondary smoking, or another exogenous source of acetonitrile such as automobile exhaust fumes). Acetonitrile has a long residence time in the body in comparison to other smoking-related compounds, 
such as benzene and acetonitrile, with concentrations in the breath of smokers prevented from further smoking taking almost one week to return to non-smoker levels (Jordan et al., 1995). For compounds other than acetonitrile, the median concentrations are reported for the total sample size $(n=68)$ as differences between smoking populations were not observed. For example, in this study, no difference was found between breath isoprene concentrations in non-smokers, ex-smokers and current smokers (respective medians: $189 \mathrm{ppbV}, 173 \mathrm{ppbV}, 165 \mathrm{ppbV}, p=0.37$ ), as was similarly found by Kushch et al. (2008).

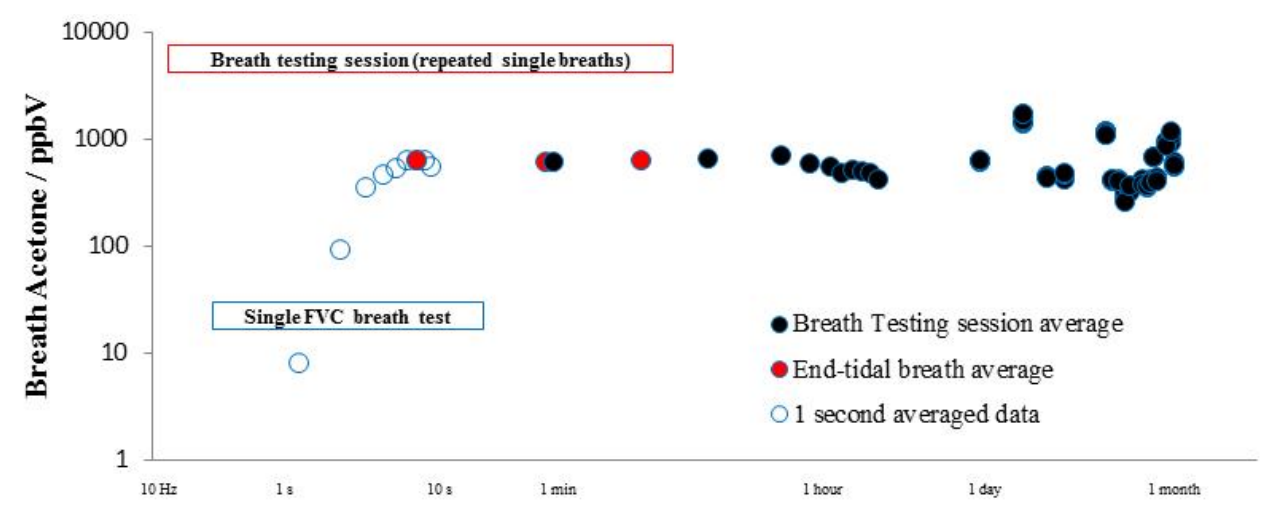

Figure 4. Longitudinal study of acetone in breath based upon repeated measurements of a 24 year-old female subject. (Open dots represent real-time acquired data; red filled dots represent end-exhaled averaged data and filled black dots represent data averaged over 3 successive breaths during a breath testing session).

Having established, based on these findings and an ever growing body of supporting literature, the spread across a population for an identified biomarker, it is necessary to examine variability over time; both across the time frame of a single direct breath measurement and breath sampling session, and over longer time frames (of particular significance with respect to a biomarker's suitability as a diagnostic tool). By excluding the dead-volume and optimising the breath sampling technique, the spread in variability during a direct breath measurement can be minimised: the end-exhaled averaged method described above typically yielded an RSD in breath compound concentration of less than $2 \%$. Over three successive breath measurements, the RSD was typically less than 5\%. An interesting example of a compound that exhibited a larger degree of variation was isoprene with an RSD of typically around $20 \%$, consistent with previous findings as isoprene has been found to respond very sensitively to changes in pulmonary ventilation and perfusion (King et al., 2009). Figure 4 shows the variation in acetone levels in the breath of a single person over the course of a day; over this timeframe the geometric standard deviation of the distribution in acetone concentration is 1.01 . 
This result is clearly lower than the inter-population spread displayed in Figure 3 over the timeframe of a single day, a finding supported by indirect PTR-MS measurements taken by Thekedar et al. (2009), who also investigated variation over timeframes of $1 \mathrm{~min}$ and 1 hour. Further, Thekedar et al. (2009) also investigated the spread over 70 days and found the variation to be much larger, a finding supported here based upon daily measurements made by an individual female subject for 4 weeks over the course of this study, resulting in a GSD of 1.5. In all cases, the intra-population variability GSD was smaller than that of the inter-population variability $(G S D=1.6)$ again, this may be an important consideration when examining the potential use of acetone as a biomarker.
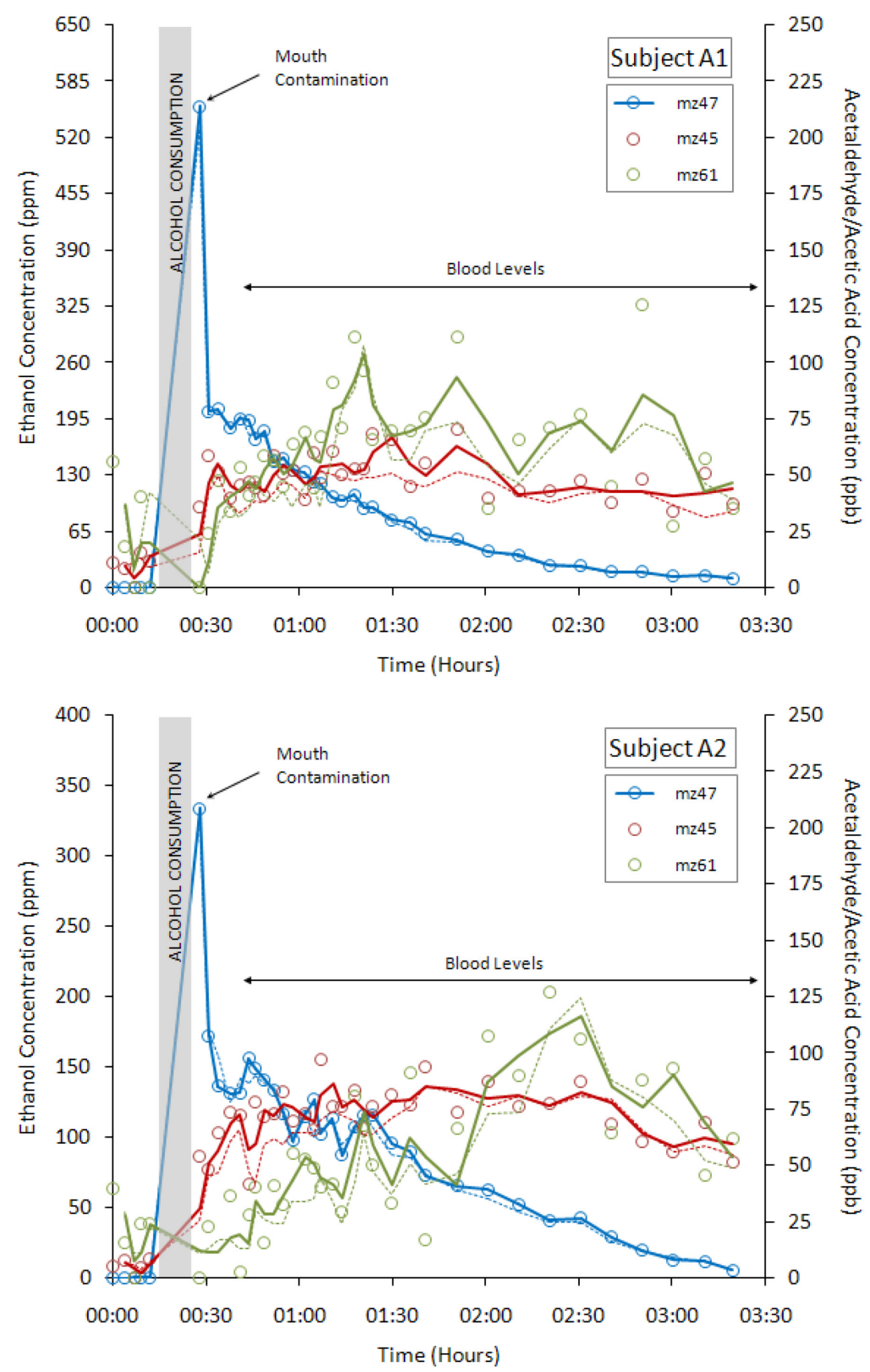
Figure 5.

Ethanol metabolism in Subjects A1 and A2 (top plot $=$ subject A1/male, bottom plot $=$ subject $\mathrm{A} 2 /$ female). The solid line links adjoining points (for $\mathrm{m} / \mathrm{z} 47$ ) or represents a 2-point moving average (for $\mathrm{m} / \mathrm{z} 45$ and $\mathrm{m} / \mathrm{z} 61$ )

\subsection{Pharmokinetics of ethanol metabolism measured on breath}

Figures 3 and 4 display evidence for the potential of PTR-MS as a diagnostic tool using a biomarker that has been previously identified and well characterised in the literature: acetone. The minimal spread in biomarker concentration over the timeframe of a session and within a single day illustrates another use of single compound analysis, namely in pharmacokinetic studies. For example such an approach could be used to track metabolites through time and monitoring patients following intervention. Several pharmacokinetic studies have already been conducted using real-time mass selective techniques; for example, Beauchamp et al. (2010) measured breath following the consumption of Eucalyptol using direct MS and Beauchamp (2011) has outlined the potential of breath measurements in pharmacokinetic studies. To exemplify this ability in the current set-up, Figure 5 shows the effect of two adults consuming two units of alcohol (50 $\mathrm{mL}$ at $40 \% \mathrm{Vol}$.). The assumption here is that the protonated parent ions represent metabolites formed as the body processes alcohol. As one would expect $m / z 47$, protonated ethanol, rises quickly. A rise in $m / z 45$, which correlates with the ethanol profile, corresponds to protonated acetaldehyde, a known intermediate of ethanol metabolism (Freund, 2000). In Figure 5 it should be noted that, whilst the sensitivity of ethanol based on $m / z=47$ is very low (see section 2.2), the high oral concentrations of ethanol encountered may exceed the linear response range of PTR-MS. Acetaldehyde is further metabolised by alcohol dehydrogenase to acetic acid. After 100 minutes, an increase in $m / z 61$ is seen, which may be protonated acetic acid levels beginning to rise as the levels of ethanol and acetaldehyde decrease. High initial ethanol concentrations were recorded owing to mouth contamination, which fall as the ethanol is cleared from the mouth. Readings then rose again as ethanol passed into the blood stream and therefore concentrations began to increase on breath (also reported by Smith et al. 2002). High levels of methanol were also recorded as the high levels of alcohol in the body inhibited metabolic loss, a result similar to that found by Lindinger et al. (1998).

As $m / z$ values obtained using PTR-MS are not strictly compound identifiers, experiments such as this breath alcohol trial, where the metabolic pathways and products are known, provide a means to support peak assignments verified by comparing results taken using different instruments. 


\subsection{Direct PTR-ToF-MS applied to fingerprinting breath}

A small trial study was conducted to test the potential clinical application of direct-ToF-MS with tidal breath collection for a group of cystic fibrosis children in order to try and identify bacterial lung infection. A total of 10 cystic fibrosis patients ( 5 male, 5 female, mean age 14) took part and 4 healthy children ( 3 male, 1 female, mean age 9) formed the control group. Microbiological results (as described in Section 2.3) confirmed bacterial or fungal infection in six of the patients.

On qualitative assessment of the results, the common breath components at $m / z 33,59,63$ and 69 (protonated methanol, acetone, dimethyl sulfide and isoprene, respectively) generally showed no trend across the patient and control groups. Average values for acetone and dimethyl sulfide were comparable to that observed in healthy adults (displayed in Table 1) whilst average methanol and isoprene values in the study subjects were generally lower than that observed in the group of healthy adults (consistent with a previous study on children's breath (Taucher et al., 1997)). Previous reports have linked dimethyl sulfide and isoprene to cystic fibrosis and respiratory exacerbation (Barker et al., 2006; Kamboures et al. 2005; McGrath et al., 2000). However, the present results indicate no significant difference between dimethyl sulfide and isoprene in the cystic fibrosis (CF) and healthy control (N) groups (mean dimethyl sulfide: $\mathrm{CF}=965 \mathrm{ncps}$ (normalized counts per second, relative to $1 \times 10^{6}$ counts of hydronium) vs. $\mathrm{N}=831 \mathrm{ncps}, p=0.28$ and mean isoprene: $\mathrm{CF}=393 \mathrm{ncps}$ (excluding outlier) vs. $\mathrm{N}=493 \mathrm{ncps}, p=0.13$ ), although conclusive results are difficult to draw from such a small sample size. For $P$. aeruginosa infection, the propanol and hydrogen cyanide content of breath samples from cystic fibrosis subjects have been examined owing to their previously identified link with cystic fibrosis (Wang et al., 2006; Enderby et al., 2009). Throughout this trial the signals in the corresponding mass channels never exceeded ambient levels in line with no identified $P$. aeruginosa infection.

Direct ToF-MS data contains many variables (approximately $40 \mathrm{~m} / z$ values for were significantly elevated above background recorded levels) for each sample so principal component analysis (PCA) was used to reduce the dimensionality of the data and to look for underlying trends. The resulting PCA biplot is shown in Figure 6. The plot presents the scores and loadings for the first principal component, describing the greatest variation in the data, against the third principal component as this provided the best separation of the groups. The PCA plot shows good but not complete separation. Healthy subjects and non-infected cystic fibrosis were not distinguishable and grouped together, but were separated from the infected cystic fibrosis subjects with the exception of one non-infected cystic fibrosis patient (whose breath profile appeared closer to the infected patients than the other noninfected patients). Interestingly, loadings representing $\mathrm{m} / \mathrm{z} 89$ and 71 in Figure 6, were also found to be elevated in bacterial culture headspace mass spectra of $S$. aureus (data not presented). 
The need for rigorous validation of findings based on multivariate analyses in breath biomarker research is discussed by Miekisch et al. (2012) and, accordingly, it is difficult to draw strong conclusions from the correlations observed in this small-scale study. Clearly, a larger data set is needed to properly investigate the observed trends, but the results highlight important areas of focus for future work in multichannel measurements of breath. Furthermore, these tests compliment recent findings whereby breath volatiles measured by secondary electrospray ionization-MS confirmed the presence of bacterial lung infection in the breath of mice (Zhu et al., 2013). Coupling direct-ToF-MS data with a statistical technique such as PCA illustrates the use of multiple variables to 'fingerprint' clinical conditions.

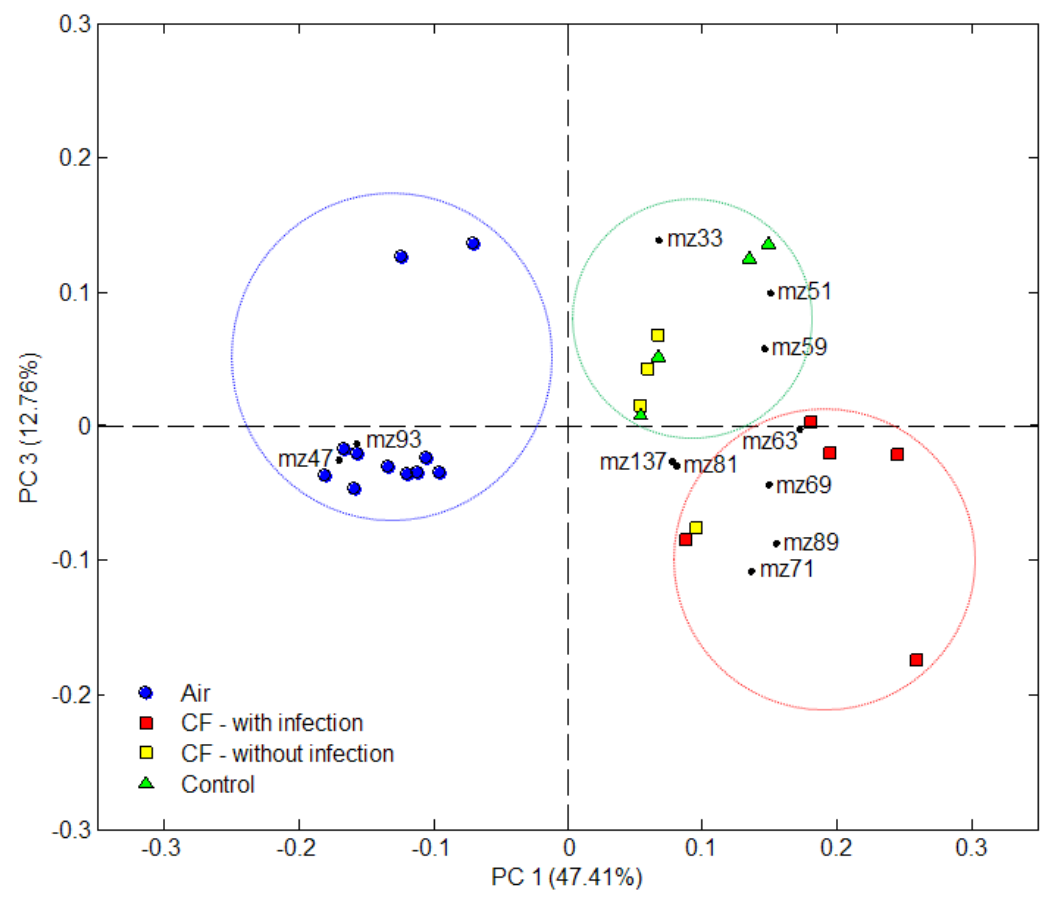

Figure 6. PCA biplot for the $1^{\text {st }}$ and $3^{\text {rd }}$ principal components for a small group of cystic fibrosis patients with and without infection $v s$ a control patient group. 


\section{Conclusion and future perspectives}

The case studies presented in this paper add to a substantial body of literature on breath studies using direct MS techniques which have built upon pioneering work using SIFT-MS (eg, Spanel and Smith, 2011) and PTR-MS (eg., Lindinger et al., 1998). The data in Figures 3 to 5 support previously published results that cover some of the background knowledge required to fully evaluate the feasibility in using direct ToF-MS in diagnostic medicine. They also represent the first time these tests have been reported using a PTR-ToF-MS technique. The data in Figure 3 outlines the spread in population variability for biomarkers measured in breath whilst Figure 4 provides an example of how a single person's breath varies over time for a single biomarker. Both of these observations are of course key to quantifying the differences between biomarkers that relate to different populations and disorders. However, compounds that show potential as diagnostic biomarkers are by their nature common in healthy breath samples and vary depending on many factors (as described above). Figure 4 , for example, shows the extent to which acetone concentrations can vary in healthy control subjects and several outliers were recorded in Figure 3 that approached levels previously recorded for uncontrolled diabetic patients (Smith and Spanel, 1999). Whilst variation in biomarker concentration between subjects and over long time frames can be large, the variance between mass channels within mass spectra may enable further diagnostic capability. In this case, diseases would be best described by a breath pattern or 'fingerprint'. This concept has been employed in analogous studies of, for example, direct headspace sampling of fungal and microbial air (for example, see O'Hara and Mayhew, 2009). In examples such as these, headspace sampling does not share the same timerestraint as online-breath sampling (enabling full mass scans to be performed by quadrupole-based systems). However, these direct techniques have not been employed to profile VOC patterns in online breath samples. Herbig et al (2009) first described the use of PTR-ToF-MS in sampling online breath and presented here, as a proof of principle, are the first measurements that use this technique to profile the breath fingerprints of children with cystic fibrosis, with and without infection.

Typically run with a cycle time equivalent to a mass range of $300 \mathrm{Da}$, a breath measurement taken by PTR-ToF-MS can provide many variables which can be processed by any number of the multivariate statistical techniques that are currently available. In using multivariate statistics, deconvolution of mass spectra is possible based upon knowledge of fragmentation patterns of breath compounds, as well as their sensitivity towards PTR-MS detection. However, deconvolution is not necessary for an electronic nose approach to successfully recognise different breath patterns. A multidimensional statistical approach was taken by Fedrigo (2010) in a breath study on ionizing radiation exposure using an offline PTR-MS approach. The results from studies such as these can be applied to PTR detection problems in general. 
Potentially, PTR-ToF-MS offers other modes of operation that have perhaps not yet been fully exploited in breath research. By standardising the breath sampling approach, the variability between successive breaths has been shown to be minimal (Thekedar et al, 2009). In this case different reagents could be used to generate different CIR spectra for successive breaths within a breath sampling session (this is the principle behind SIFT-MS (Smith and Spanel, 2010)). The ability of different reagent ions to distinguish between isobaric species has already been established in PTRToF-MS (Wyche et al., 2005) and the use of alternative reagent ions to $\mathrm{H}_{3} \mathrm{O}^{+}$such as $\mathrm{O}_{2}^{+}$and $\mathrm{NO}^{+}$, would generate different spectral patterns, thereby widening the number of variables to be analysed using a multidimensional statistical approach (Blake et al, 2006).

Finally, as near real-time analysis enables the capture of a full breath cycle, it is possible to apportion different breathing phases in an equivalent manner to the procedures used in spirometry and capnography. The statistics generated by this process may also provide metrics for multivariate analysis that could yield further diagnostic potential. The sensitivities reported by Wyche et al. (2008) in one of the earliest reported PTR-ToF-MS experiments were of the order 0.1 counts $\mathrm{ppbV}^{-1} \mathrm{~s}^{-1}$ per compound. Based upon standard requirements for spirometry, measurements should be taken on a 0.2 s timeframe (Miller et al. 2008), which would therefore yield an approximate limit of detection of 9 $\mathrm{ppbV}$. For many compounds, this would be too insensitive to observe VOCs on breath. However, recent technological developments have drastically improved these detection limits; Herbig et al. (2009) report a system that is both highly mass-resolved and sensitive whilst Barber et al. (2012) have recently reported the development of a radio frequency (RF) ion funnel capable of increasing sensitivity by two orders of magnitude in PTR-ToF-MS systems. These technological advances make the inclusion of dynamic statistics in multivariate breath analysis possible in the near future. 


\section{References}

Abbott S M, Elder J B, Španěl P and Smith D 2003 International J. Mass Spec 228(2-3) 655-665

Allardyce R A, Langford V S, Hill A L and Murdoch D R 2006 J. Microbiol. Methods (2) 361-365

Amann A, Poupart G, Telser S, Ledochowski M, Schmid A and Mechtcheriakov S 2004 Int. J. Mass Spec. 239 227-233

Amann A, Miekisch W, Pleil J, Risby T, Schubert J 2010 Eur. Respir Min. 49 96-114

Barber S, Blake R S, White I R, Monks P S Reich F, Mullock S and Ellis A M. 2012 Anal. Chem. 84 $5387-5391$

Barker M, Hengst M, Schmid J, Buers H J, Mittermaier B, Klemp D, Koppman R 2006 Eur. Respir. J 27(5) 929-936

Baumbach J I 2009 J. Breath Res, 3 1-1

Beauchamp J, Herbig J, Gutmann R and Hansel A 2008 J. Breath Res. 2046001

Beauchamp J, Kirsch F and Buettner A 2010 J. Breath Res. 4(2) 026006

Beauchamp J 2011 J. Breath Res. 5037103

Blake R S, Whyte C, Hughes C O, Ellis A M, Monks P S 2004 Anal. Chem. 76 3841-3845

Blake R S, Monks P S, Ellis A M 2009 Chem. Rev. 109 861-896

Blake R S, Wyche K P, Ellis A M, Monks P S 2006 Int. J. Mass. Spectrom., 254 85-93

Blake R S, Monks P S, Ellis A M 2012 Spectroscopy in Europe, 24, 17-20

Boots A W, Berkel J J B N, Dallinga J W, Smolinska A, Wouters E F and Schooten F J $2012 \mathrm{~J}$. Breath Res. 6027108

Brunner C, Szymczak W, Höllriegl V, Mörtl S, Oelmez H, Bergner A, Huber R M, Hoeschen C and Oeh U 2010 Anal. Bioanal. Chem. 396(6) 2315-2324

Carroll W, Lenney W, Wang T S, Španěl P, Alcock A and Smith D 2005 Pediatric Pulmonology 39(5) 452-456

Deng C H, Zhang J, Yu X F, Zhang W and Zhang X M 2004 J. Chrom. B 810(2) 269-275

Di Natale C, Macagnano A, Martinelli E, Paolesse R, D'Arcangelo G, Roscioni C, Finazzi-Agro A and D'Amico A 2003 Biosensors and Bioelectronics 18 1209-18

Dragonieri S, Schot R, Mertens B J, Le Cessie S, Gauw S A, Spanevello A, Resta O, Willard P, Vink T J, Rabe K F, Bel E H and Sterk P J 2007 J. Allergy Clin. Immun, 120(4) 856-862

Dweik R A, Boggs P B, Erzurum S C, Irvin C G, Leigh M W, Lundberg J O, Olin A, Plummer and Taylor D R 2011 Am. J. Respir. Crit. Care Med. 184602 - 615 
Enderby B, Smith D, Carroll W, Lenney W 2009 Pediatric Pulmonology 44(2) 142-147

Fedrigo M, Hoeschen C and Oeh U 2010 Int. J. Mass. Spec. 29513 - 20

Gardner J W, Shin, H W and Hines E L 2000 Sensors and Actuators B: Chemical 70 19-24

Herbig J, Titzmann T, Beauchamp J,Kohl L and Hansel A 2008 J. Breath Res. 2037008

Herbig J, Müller M, Schallhart S, Titzmann T, Graus M and Hansel A 2009 J. Breath Res. 3027004

Jansson B O and Larsson B T 1969 J. Lab. Clin. Med. 74 961-966

Jordan A, Haidacher S, Hanel G, Hartungen E, Märk L, Seehauser H, Schottkowsky R, Sulzer P and Märk T D 2009 Int. J. Mass Spec. 286 122-128

Jordan A, Hansel A, Holzinger R and Lindinger W 1995 Int. J. Mass Spec. and Ion Proc. 148 (1-2) L1-L3

Lindinger W, Jordan A 1998 Chem. Soc. Rev. 27 347-375

Kamboures M S, Blake D R, Cooper D M, Newcomb R L, Barker M, Larson J K, Meinardi S, Nussbaum E, Rowland F S 2005 Proceedings of the National Academy of Sciences 102(44) 1576215767

Keck L, Hoeschen C and Oeh U 2008 Int. J. Mass. Spec. 156 - 165

Kim K H, Shamin Ara Jahan and Ehsanul Kabir 2012 Trends Anal. Chem. $331-8$

King J, Kupferthaler A, Unterkofler K, Koc H, Teschl S, Teschl G, Miekisch W, Schubert J, Hinterhuber H and Amann A 2009 J. Breath Res. 3027006

King J, Mochalski P, Kupferthaler A, Unterkofler K, Koc H, Filipiak W, Teschl S, Hinterhuber H and Amann A 2010 Physiol. Meas. 31(9) 1169-84

Kushch I, Arendacká B, Stolc S, Mochalski P, Filipiak W, Schwarz K, Schwentner L, Schmid A, Dzien A, Lechleitner M, Witkovsky V, Miekisch W, Schubert J, Unterkofler K and Amann A 2008 Clin. Chem. Lab. Med. 46 (7) 1011-1018

Lechner M, Fille M, Hausdorfer J, Dierich M, Rieder J 2005 Current Microbiol. 51(4) 267-269

McGrath L T, Patrick R, Mallon P, Dowey L, Silke B, Norwood W, Elborn S 2000 Eur. Respir. J, 16(6) 1065-1069

Miekisch W, Schubert J K and Noeldge-Schomburg G F E 2004 Clin. Chim. Acta 347 25-39

Miekisch W, Herbig J and Schubert J K 2012 J. Breath Res. 6036007

Miller M R, Hankinson J, Brusasco V, Burgos F, Casaburi R, Coates A, Crapo R, Enright P, van der Grinten CP, Gustafsson P, Jensen R, Johnson DC, MacIntyre N, McKay R, Navajas D, Pedersen OF, Pellegrino R, Viegi G, Wanger J; ATS/ERS Task Force 2005 Eur. Respir. J. 26(2) 319-38 
O’Hara, O’Hehir S O, Green S and Mayhew C A 2008 Physiol. Meas. 29 309-330

O’Hara M, Mayhew C A 2009 J. Breath Res. 3027001

Phillips M, Gleeson K, Hughes J M, Greenberg J, Cataneo R N, Baker L and McVay W P 1999a Lancet 353 1930-3

Phillips M, Herrera J, Krishnan S, Zain M, Greenberg J, Cataneo R N 1999b J. Chrom. B 729 75-88

Ruzsanyi V and Baumbach J I 2005 Int. J. Ion Mobility Spectrom 8 5-8

Scotter J M, Langford V S, Wilson P F, McEwan M J and Chambers S T 2005 J. Microbiol. Methods 63 (2) $127-134$

Scotter J M, Allardyce R A, Langford V, Hill A and Murdoch D R 2006 J. Microbiol. Methods 65(3) $628-631$

Smith D, Španěl P and Davies S 1999 J. Appl. Physiol. 87(5) 1584-1588

Smith D, Wang T S and Španěl P 2002 Physiol. Measurement 23(3) 477-489

Smith D and Spaněl P 2004 Mass Spectrom. Rev. 24 661-700

Smith D, Turner C and Španěl P 2007 J. Breath Res. 1(1) 1-12

Spaněl P and Smith D 2011 Mass Spectrom. Rev. 30 236-67

Steeghs M M L, Cristescu S M, Munnik P, Zanen P and Harren F J M 2007 Physiol. Meas. 28 73-84

Schwarz K, Filipiak W and Amann A 2009 J. Breath Res. 3027002

Tassopoulos, C N, Barnett D and Russell Fraser T 1969 Lancet 7609 1282-6

Taucher J, Hansel A, Jordan A, Lindinger W 1996 J. Agricultural Food Chem. 44(12) 3778-3782

Taucher J, Hansel A, Jordan A, Fall R, Futrell J H and Lindinger W 1997 Rapid Commun. Mass Spectrom. 11(11) 1230-1234

Thekedar B, Szymczak W, Höllriegl V, Hoeschen C and Oeh U 2009 J. Breath Res. 3027007

Thorn R M, Greenman J 2012 J. Breath Res. 6(2):024001

Turner C, Španěl P and Smith D 2006 Physiological Measurement 27(4) 321-337

Ueta I, Saito Y, Hosoe M, Okamoto M, Ohkita H, Shirai S, Tamura H, Jinno 2009 J. Chrom. B 77(24) 2551-2556

Wang T S, Carroll W, Lenny W, Boit P, Smith D 2006 Rapid Commun.Mass Spectrom. 20(2) 125130 
Wyche K P, Whyte C, Blake R S, Willis K A, Monks P S and Ellis A M, 2005 Rapid Commun. Mass Spectrom. 19 3356-3362

Wyche K P, Blake R S, Ellis A M, Monks P S, Brauers T, Koppmann R and Apel E C 2007 Atmos. Chem. Phys. 7 609-620

Wyche K P, Whyte C, Blake R S, Willis K A, Ellis A M and Monks P S 2008 Adv. Env. Monitoring 1 64-76

Zhu J, Bean H D, Wargo M J, Leclair L W and Hill J E 2013 J. Breath Res. 7016003 